\title{
Evidence of reciprocal reorientation of the catalytic and hemopexin-like domains of full-length MMP-12
}

\author{
I. Bertini, ${ }^{1,2}$ V. Calderone, ${ }^{1}$ M. Fragai, ${ }^{1,3}$ R. Jaiswal, ${ }^{1}$ \\ C. Luchinat ${ }^{1,3}$ and M. Melikian ${ }^{1}$ \\ ${ }^{1}$ Magnetic Resonance Center (CERM) - University of Florence, Via L. Sacconi 6, 50019 Sesto Fiorentino, Italy \\ 2 Department of Chemistry - University of Florence, Via della Lastruccia 3, 50019 Sesto Fiorentino, Italy \\ ${ }^{3}$ Department of Agricultural Biotechnology, University of Florence, Via Maragliano, 75-77, 50144 Florence, Italy
}

Matrix metalloproteinases (MMP) are an important family of 23 proteins which are involved in a number of extracellular processes including the degradation of the extracellular matrix. ${ }^{1,2}$ All active MMPs but MMP-7 are constituted by two domains, a catalytic (CAT) and a hemopexin-like (HPX) domain. The CAT and HPX domains are connected by a linker whose length varies from 14 to 68 AA. $^{3,4}$ For the majority of MMPs the linker is relatively short (14-23 AA) while for MMP-9 and MMP-15, at the other extreme, the intervening residues between the CAT and HPX domains (63 and $68 \mathrm{AA}$, respectively), constitute a further, highly glycosylated, domain termed OG domain. The CAT domain alone bears full proteolytic activity towards a range of peptides and proteins. However, efficient proteolysis of, for instance, triple helical collagen requires the full-length active protein. For this reason it is often hypothesized that the HPX domain helps the local unwinding of the triple helix, in such a way that a single peptide strand can be accommodated in the active site of the CAT domain and cleaved. It has been also hypothesized that a relative mobility of the HPX domain is necessary for this function. In case of full-length MMP-12 the low resolution crystal structure we collected (Figure 1) was largely sufficient to establish that the structure is less compact, and the relative orientation of the two domains totally different, with respect to the four Xray structures of MMP-1 and MMP-2 already described.
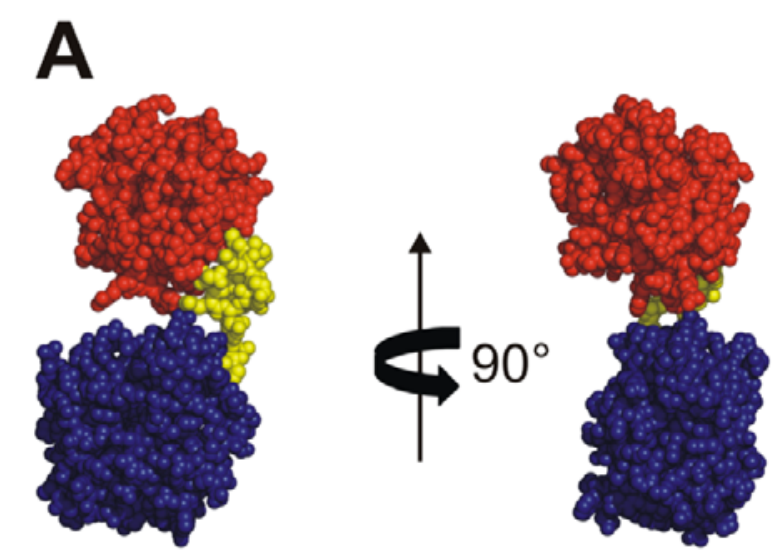

Figure 1: Space-filling representations of the X-ray structures of FL-MMP-12.

Moreover NMR data collected in solution show that the two domains are not held rigidly to one another, but must undergo independent motions. Therefore SAXS analysis has been performed in order to investigate the types and variety of the sampled conformations. Synchrotron X-ray scattering data from FL-MMP-12 solutions was collected on the X33 beamline, of the EMBL (DESY, Hamburg), using a MAR345 image plate detector. The scattering patterns were measured with a 2 minute exposure time for several solute concentrations in the range from 0.8 to $8.3 \mathrm{mg} / \mathrm{ml}$. The processed X-ray scattering pattern from FL-MMP-12 presented in Figure 1 yields a molar mass 
estimate of $40 \pm 4 \mathrm{kDa}$, compatible with that calculated from the sequence ( $42.5 \mathrm{kDa})$, indicating that the protein is monomeric in solution. The experimental radius of gyration $\mathrm{R}_{\mathrm{g}}$ and maximum size $D_{\max }$ are $31 \pm 1 \AA$ and $110 \pm 10 \AA$, respectively. These values significantly exceed the parameters calculated from the X-ray structure of FL-MMP-1 $\left(\mathrm{R}_{\mathrm{g}}=25 \AA, \mathrm{D}_{\max }=85 \AA\right)$, while they are in better agreement with those computed from the less compact X-ray structure of FL-MMP-12 $\left(\mathrm{R}_{\mathrm{g}}=29 \AA\right.$, $\mathrm{D}_{\max }=95 \AA$ ). The scattering curve computed from the present FL-MMP-12 structure displays a much better agreement to the experiment $(\chi=2.5$, curve (3) in Figure 2$)$, but still display some systematic deviations.

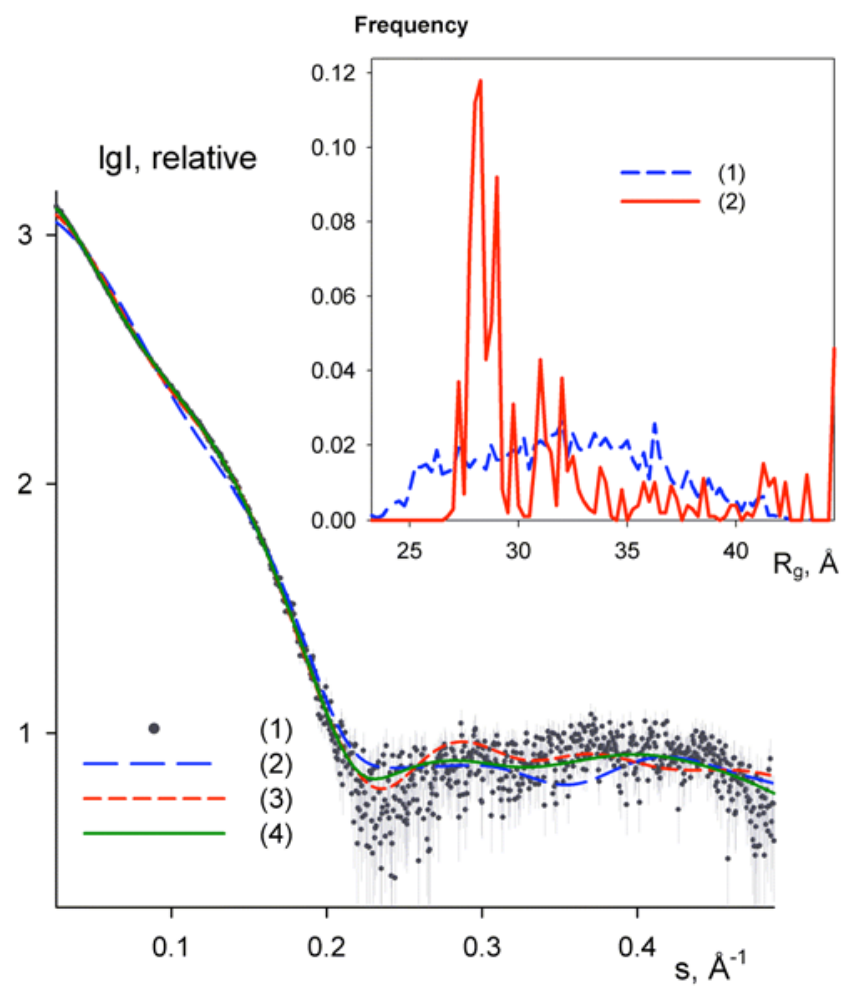

Figure 6: Experimental X-ray scattering from FL-MMP-12 and scattering from the models. (1) experimental data with error bars; (2-3) computed scattering from the crystallographic models of FL-MMP-1 and FL-

MMP-12, respectively; (4) a typical fit by the selected ensemble of structures. The logarithm of the scattering intensity is plotted against the momentum transfer. Insert: the frequency of the models with the

given $\mathrm{R}_{\mathrm{g}}$ in the initial pool of structures with randomized interdomain linkers (1) and in the selected ensembles (2); the latter distribution is obtained by the averaging of several EOM runs. Both $\mathrm{R}_{\mathrm{g}}$ distributions are normalized to the integral value of unity.

The EOM analysis indicates that the present crystal structure of FL-MMP-12 may be significantly present also in solution, but also that the protein reveals noticeable conformational flexibility, as revealed by the presence of a more or less uniform distribution of particles in the range between $\mathrm{R}_{\mathrm{g}}$ $=30$ and $50 \AA$ and a significant spike, which was always observed for the most extended particles (Figure 2, insert). The collected data demonstrate that full-length MMP-12 shows relative mobility of its catalytic and hemopexin domains and open new perspectives for the often hypothesized active role of the hemopexin-like domain in the enzymatic activity of MMPs.

\section{References}

(1) W.C. Parks; C.L. Wilson, and Y.S. Lopez-Boado, Nat.Rev.Immunol. 2004, 4, 617-629.

(2) A. Page-McCaw, A. Ewald, Z. Werb, Nat.Rev.Mol.Cell Biol. 2007, 8, 221-233.

(3) K. Maskos, W. Bode, Mol.Biotechnol. 2003, 25, 241-266.

(4) C. Andreini, L. Banci, I. Bertini, C. Luchinat, A. Rosato, J.Proteome Res. 2004 , 3, 21-31. 